\section{(6) OPEN ACCESS}

\title{
Physical frailty and pulmonary rehabilitation in COPD: a prospective cohort study
}

\author{
Matthew Maddocks, ${ }^{1}$ Samantha S C Kon, ${ }^{2,3}$ Jane L Canavan, ${ }^{2,4}$ Sarah E Jones, ${ }^{2,4}$ \\ Claire M Nolan, ${ }^{2,4}$ Alex Labey, ${ }^{2,4}$ Michael I Polkey, ${ }^{2}$ William D-C Man ${ }^{2,4}$
}

\begin{abstract}
- Additional material is published online only. To view please visit the journal online (http://dx.doi.org/10.1136/ thoraxjnl-2016-208460)
\end{abstract}

${ }^{1}$ King's College London, Cicely Saunders Institute, London, UK ${ }^{2}$ NIHR Respiratory Biomedical Research Unit, Royal Brompton \& Harefield NHS Foundation Trust and Imperial College, London, UK

${ }^{3}$ The Hillingdon Hospital NHS Foundation Trust, Middlesex, UK

${ }^{4}$ Harefield Pulmonary Rehabilitation Unit, Harefield Hospital, Middlesex, UK

Correspondence to: Dr Matthew Maddocks, King's College London, Cicely Saunders Institute, London SE5 9PJ, UK:

matthew.maddocks@kcl.ac.uk

Received 8 February 2016 Revised 27 April 2016

Accepted 6 May 2016 Published Online First 13 June 2016

\begin{abstract}
Background Frailty is an important clinical syndrome that is consistently associated with adverse outcomes in older people. The relevance of frailty to chronic respiratory disease and its management is unknown. Objectives To determine the prevalence of frailty among patients with stable COPD and examine whether frailty affects completion and outcomes of pulmonary rehabilitation.

Methods 816 outpatients with COPD (mean (SD) age $70(10)$ years, FEV $\%$ predicted $48.9(21.0))$ were recruited between November 2011 and January 2015. Frailty was assessed using the Fried criteria (weight loss, exhaustion, low physical activity, slowness and weakness) before and after pulmonary rehabilitation. Predictors of programme non-completion were identified using multivariate logistic regression, and outcomes were compared using analysis of covariance, adjusting for age and sex.
\end{abstract}

Results $209 / 816$ patients $(25.6 \%, 95 \%$ Cl 22.7 to 28.7) were frail. Prevalence of frailty increased with age, Global Initiative for Chronic Obstructive Lung Disease (GOLD) stage, Medical Research Council (MRC) score and age-adjusted comorbidity burden (all $p \leq 0.01$ ). Patients who were frail had double the odds of programme non-completion (adjusted OR 2.20, 95\% Cl 1.39 to $3.46, p=0.001)$, often due to exacerbation and/ or hospital admission. However, rehabilitation outcomes favoured frail completers, with consistently better responses in MRC score, exercise performance, physical activity level and health status (all $p<0.001$ ). After rehabilitation, $71 / 115(61.3 \%)$ previously frail patients no longer met case criteria for frailty.

Conclusions Frailty affects one in four patients with COPD referred for pulmonary rehabilitation and is an independent predictor of programme non-completion. However, patients who are frail respond favourably to rehabilitation and their frailty can be reversed in the short term.

\section{INTRODUCTION}

Frailty describes a clinical syndrome characterised by multisystem decline that leads to reduced functional reserve and increased vulnerability to dependency or mortality following minor stressor events. ${ }^{1}$ It affects an estimated 1 in every 10 people aged over 65 years $^{2}$ and is consistently associated with increased risk of falls, disability, hospitalisation and death. ${ }^{3}$ Although frailty is conventionally considered secondary to age-related decline, chronic disease(s) can accelerate the rate of decline and

\section{Key questions}

What is the key question?

What is the prevalence of frailty in stable COPD, and does frailty affect the completion and outcomes of pulmonary rehabilitation?

\section{What is the bottom line?}

- Frailty affects one in every four patients with COPD entering pulmonary rehabilitation, is associated with favourable outcomes, but is also strong risk factor for non-completion.

\section{Why read on?}

- This is the first characterisation of the frailty phenotype in stable COPD and demonstrates that physical frailty is amenable to treatment with pulmonary rehabilitation.

precipitate a frail state. In COPD, extrapulmonary manifestations include physical inactivity, muscle weakness, anorexia, osteoporosis and fatigue. ${ }^{4}$ Each of these systemic impacts of the disease is frequently observed in physical frailty.

The relevance of frailty to chronic respiratory disease has not been fully dissected. In retrospective cohort studies, self-reported frailty is more common in older people with COPD than without it, and markers of frailty have identified those at increased risk of subsequent hospital admission or death. ${ }^{5}$ Few studies have examined the prevalence of frailty in respiratory disease using validated definitions. One notable exception is a report restricted to lung transplant candidates, among whom frailty was associated with increased risk of delisting or death. ${ }^{7}$ Identifying frailty earlier in the course of disease is important, as interventions may then be introduced to prevent functional decline, hospital admissions and/or death in those at high risk. Frailty may prove a valuable way of stratifying patients with COPD for future management as it accounts for multiple deficits that influence disease prognosis, for example, muscle weakness or physical inactivity, ${ }^{8}$ including deficits not considered by other syndromes or comorbidity indices. ${ }^{9} 10$

Another important but unstudied topic is the interplay between frailty and pulmonary rehabilitation. Pulmonary rehabilitation is highly effective at improving symptom burden, physical function and 
health status, although patient response is heterogeneous. ${ }^{11} 12$ Conceptually, pulmonary rehabilitation targets many components of frailty, including slowness, ${ }^{13}$ fatigue, ${ }^{14}$ weakness ${ }^{15}$ and physical inactivity, ${ }^{11}$ and provides a holistic approach to encourage self-management, disease education and behaviours to improve overall health. Pulmonary rehabilitation also targets dyspnoea, which may be a contributing factor to the development of frailty in people with chronic respiratory disease. Finally, some programmes incorporate falls prevention strategies, through balance training and education, ${ }^{16}$ again focusing on a frailty-related outcome. However, in the same way that frailty affects planned surgical management, ${ }^{7}$ the syndrome may prevent patients from engaging in pulmonary rehabilitation-a mainstay of disease management. ${ }^{11}$ The factors for heterogeneous adherence to pulmonary rehabilitation are widely debated, ${ }^{17}{ }^{18}$ but physical frailty is a plausible candidate given the close relationship with adverse outcomes in $\operatorname{COPD}^{6} 7$ and other long-term conditions. ${ }^{3}$ If frailty does hinder completion of pulmonary rehabilitation, this would suggest a need to support patients who are frail with alternative or supplementary rehabilitation strategies.

Study objectives were to determine the prevalence of frailty among patients with stable COPD, describe the clinical characteristics of the COPD frailty phenotype and examine whether frailty affects completion and clinical outcomes of pulmonary rehabilitation. We hypothesised that frailty would independently predict non-completion of pulmonary rehabilitation.

\section{METHODS}

\section{Participants and design}

Participants were recruited to this prospective cohort study from respiratory outpatient and pulmonary rehabilitation clinics at Harefield Hospital (Middlesex, UK) between November 2011 and January 2015. Eligible patients were aged 35 years or above, with a physician diagnosis of COPD consistent with the Global Initiative for Chronic Obstructive Lung Disease (GOLD) criteria $^{19}$ and appropriate for a pulmonary rehabilitation referral. Exclusion criteria were an exacerbation in the previous 4 weeks that required a change in medication, a condition that might make moderate-intensity exercise unsafe, for example, unstable cardiac disease or a predominant neurological disability. Treating clinicians identified potentially eligible patients and offered a written information sheet. Referral criteria for pulmonary rehabilitation were able to walk at least $5 \mathrm{~m}$, any degree of functional impairment secondary to dyspnoea (typically Medical Research Council (MRC) dyspnoea score 2 or more), no previous supervised pulmonary rehabilitation in previous 12 months and without unstable cardiovascular disease, in line with British Thoracic Society Quality Standards. ${ }^{20}$ Data for some participants $(528 / 816,64.7 \%)$ relating to sarcopenia, but not frailty, have been reported previously. ${ }^{15}$ All participants gave written informed consent in accordance with the principles of Good Clinical Practice and the Declaration of Helsinki, and the study was approved by the West London (11/H0707/2) and London Camberwell St Giles (11/LO/1780) Research Ethics Committees.

\section{Frailty assessment}

Frailty was defined using the Fried phenotype model, ${ }^{9}$ which is well established and validated in large epidemiological studies. ${ }^{3}$ This model comprises five characteristics that reduce physiological reserve and precipitate a vulnerable state; shrinking (unintentional weight loss), exhaustion, low physical activity, slowness and weakness. ${ }^{9}$ Characteristics were assessed by weight loss history, two questions from the Center for Epidemiological Studies-Depression (CES-D) questionnaire (exhaustion), ${ }^{21}$ weekly self-reported energy expenditure using the modified Minnesota Leisure-Time Physical Activity Questionnaire (low physical activity), ${ }^{22}$ 4-m gait speed (4MGS, slowness) ${ }^{23}$ and handgrip dynamometry (weakness). Standardised criteria, derived from the original reference cohort, ${ }^{9}$ were used to define each characteristic as either present or absent for each patient (see online supplementary table S1), providing an ordinal score ranging $0-5$. Patients with no criteria present were considered not-frail/robust, those meeting 1-2 criteria were considered prefrail and those with $\geq 3$ criteria present were considered frail.

\section{Additional assessments}

Bioelectrical impedance analysis (Quadscan 4000, Bodystat, Isle of Man, UK) was used to estimate skeletal muscle mass index (SMI). ${ }^{24}$ The presence of sarcopenia was defined according to the consensus European Working Group on Sarcopenia in Older People criteria. ${ }^{25}$ Quadriceps maximum voluntary contraction (QMVC) was measured using a fixed strain gauge, ${ }^{26}$ with weakness diagnosed according to healthy predicted values ${ }^{27}$ and sexspecific functionally relevant cut-points. ${ }^{28}$ Exercise performance was assessed using the incremental shuttle walk test (ISWT). ${ }^{29}$ Further measurements included evaluation of respiratory disability by MRC dyspnoea score, comorbidity burden using the age-adjusted Charlson Index, ${ }^{30}$ help with activities of daily care using the Katz Index, ${ }^{31}$ composite disease severity by the age, dyspnoea, and airflow obstruction (ADO) index, ${ }^{32}$ Hospital Anxiety and Depression Scale (HADS) and health status using the self-reported Chronic Respiratory Questionnaire (CRQ) ${ }^{33}$ and COPD Assessment Test (CAT). ${ }^{34}$

\section{Pulmonary rehabilitation}

Pulmonary rehabilitation was an 8 -week outpatient exercise and multidisciplinary education programme, comprising two supervised and at least one additional home-based session each week and organised according to the British Thoracic Society Quality Standards for Pulmonary Rehabilitation. ${ }^{20}$ Supervised sessions comprised 1 hour of exercise and $45 \mathrm{~min}$ of education. Exercise training was individualised and progressive. Initial walking speed prescription was at $80 \%$ of predicted peak oxygen consumption based on ISWT performance, ${ }^{35}$ while initial endurance cycling was set at a workload with the aim of patients completing $10 \mathrm{~min}$ of continuous training. Lower limb resistance training comprised 2 sets of 10 seated leg press repetitions, performed with an initial training load of $60 \%$ one-repetition maximum, as well as sit-to-stand, knee extension, hip flexion and hip abduction exercises with ankle weights. Upper limb resistance training comprised biceps curls, shoulder press and upright row with free weights. Education was delivered by a multidisciplinary team. Topics were chosen to develop patients' understanding and holistic management of their disease and included physical activity and exercise, medication use, diet, smoking cessation, coping strategies, as well as managing infections through early recognition, rescue medication and appropriate general practice/hospital presentation.

\section{Statistical analysis}

Our sample size was based on the precision to which the overall prevalence of frailty could be estimated. Assuming it was within the range $10 \%-60 \%$, prevalence could be estimated to within $\pm 3.5 \%$ using 800 participants with a large sample normal approximation (nQuery Advisor V.6.0). Analyses were performed using SPSS (V.22, IBM, New York, USA) and graphs 
produced using Prism 5 (GraphPad Software, San Diego, California, USA). Data were presented as proportions (95\% CIs) or mean (SD).

The prevalence of frailty was determined overall and then compared across groups according to age, GOLD spirometry stage, MRC dyspnoea score and age-adjusted Charlson comorbidity score using $\chi^{2}$ for trend. Baseline characteristics as well as pulmonary rehabilitation uptake, attendance and completion rates were compared across groups (not frail/robust, pre-frail, frail) using one-way analysis of variance or $\chi^{2}$ for trend with a Bonferroni correction applied to post hoc pairwise comparisons. Uptake was defined as the proportion of assessed patients who attended the first supervised session, adherence was assessed using the number of supervised sessions attended and completion was defined as the proportion of patients who had documented attendance at a minimum of eight supervised sessions, representing 50\% attendance. ${ }^{36}$

Univariate logistic regression was used to assess the relationships between non-completion, frailty status (not or prefrail/ frail) and candidate explanatory variables informed from existing literature and clinical judgement. ${ }^{17}{ }^{18}$ Variables associated with non-completion $(\mathrm{p}<0.15)$ were considered in a multivariate model. After checking for collinearity $(r<0.75)$, we used a backwards conditional approach to retain variables in the model $(p<0.10)$. Outcomes of rehabilitation were summarised as change pre-to-post rehabilitation for patients who completed as per the above definition. Outcomes were then compared across groups using analysis of covariance adjusting for age and sex. To control for Type I errors in view of multiple testing, we applied a Bonferroni correction to a significance level of 0.05 when comparing baseline patient characteristics and rehabilitation outcomes.

\section{RESULTS}

Prevalence of frailty

Eight-hundred and sixteen patients took part (484 men, mean (SD) age 70 (10) years, $\mathrm{FEV}_{1} 48.9$ (21.0) \% predicted, MRC score $3.3(1.0)$ ) (table 1). The overall prevalence of frailty was $25.6 \%$ (95\% CI $22.7 \%$ to $28.7 \%$ ), while $10.0 \%$ (95\% CI $8.2 \%$ to $12.3 \%$ ) of patients did not meet any of the frailty criteria and were considered robust (figure 1). Of the frailty characteristics, the exhaustion criterion was met by the largest proportion of patients (65.3\%) followed by weakness, low physical activity and slowness. The unintentional weight loss criterion was met by fewest patients $(14.2 \%)$ (table 1$)$. Frailty tended to be more common among women than men $(29.7 \%$ vs $22.8 \%, p=0.08)$. Prevalence increased statistically with increasing age $(p<0.001)$, GOLD stage $(p=0.01)$, MRC score $(p<0.001)$ and comorbidity burden $(p=0.004)$. There was a twofold increase in frailty prevalence among patients with GOLD stage IV as compared with stage I disease $(34.7 \%$ vs $17.9 \%, \mathrm{p}<0.001)$ and a threefold increase among patients with an MRC score of 5 as compared with a score of $3(62.1 \%$ vs $21.0 \%, \mathrm{p}<0.001$; figure 2$)$.

\section{The COPD frailty phenotype}

Patients who were frail with COPD had significantly reduced SMI but not body mass index as compared with prefrail or robust patients (table 1). Reduced physical function was evident beyond the characteristics used to define frailty, with reduced QMVC and ISWT performance, and a greater prevalence of sarcopenia $(23.9 \%$ vs $9.5 \%$ in the prefrail group and $1.2 \%$ in the robust group, $\mathrm{p}<0.001)$. Patients who were frail reported a worse health status across all instruments and domains, and higher levels of anxiety and depression as compared with patients who were not-frail and prefrail (table 1). Among patients with QMVC measurements $(n=707)$, almost threequarters $(73.1 \%)$ of patients who were frail had concurrent quadriceps weakness, ${ }^{20}$ while only $25.0 \%$ had concurrent sarcopenia. Similar proportions of patients who were frail had both $(14.4 \%)$ or neither $(16.3 \%)$ of these phenotypes.

\section{Engagement in pulmonary rehabilitation}

Overall rates of programme uptake and completion for the cohort were $84.7 \%$ (95\% CI $82.0 \%$ to $87.0 \%$ ) and $70.3 \%$ (95\% CI 67.1\% to 73.4\%), respectively, and mean (SD) attendance was 11.4 (4.2) of 16 supervised sessions (table 1). Rates were lowest in the frail group such that $55.0 \%$ of candidates completed rehabilitation, as compared with $74.5 \%$ of prefrail and $84.1 \%$ of not frail/robust candidates $(p<0.001)$. In the univariate regression age, $\mathrm{MRC}$ score, $\mathrm{FEV}_{1} \%$ predicted, Charlson Index, ISWT, QMVC, ADO, CAT score, HADS anxiety and depression, and frailty status were associated with noncompletion. Each of the frailty characteristics was individually associated with non-completion though the relationship was strongest when frailty status was considered overall (table 2). In the multivariate regression, frailty status, age, ISWT and CAT score were retained in the final model. Frailty was a strong independent predictor and being frail was associated with double the odds of non-completion; adjusted OR 2.20 (95\% CI 1.39\% to $3.46 \%$ ), $\mathrm{p}=0.001$ (table 2). When examining reasons for not taking up or completing a programme, proportionally more frail patients experienced deterioration in their condition or were admitted to hospital (figure 1). Among patients who were frail, baseline MRC score was higher in non-completers as compared with completers $(4.3$ vs $3.9, \mathrm{p}=0.001)$ and there was weak evidence of additional functional impairment in non-completers (see online supplementary table S2).

\section{Outcomes of pulmonary rehabilitation}

Following completion of rehabilitation, significant improvements were observed across all groups for SMI, QMVC, CRQ dyspnoea, fatigue, emotional and mastery domains, and physical activity (table 3 ) and in prefrail and frail groups for MRC score, handgrip strength, ISWT, CAT score and HADS domains (table 3). Adjusting for age and sex, a gradient of treatment response in favour of patients who were frail was evident for MRC score, handgrip strength, ISWT, CRQ fatigue, emotional and mastery domains, CAT score and HADS scores (table 3). Outcomes related to frailty characteristics (handgrip strength, 4MGS and physical activity) and responses to CES-D exhaustion questions also improved, such that postrehabilitation, fewer patients met case criteria for frailty (figure 3). Among the 115 completers who were frail prior to pulmonary rehabilitation, 71 $(61.7 \%)$ were prefrail $(64,55.6 \%)$ or robust $(7,6.1 \%)$ following it. A small minority of completers, $13 / 390$ (3.3\%), had moved from a prefrail to a frail state (figure 3 ).

\section{DISCUSSION}

In this prospective cohort study, we identified that one-quarter (25.6\%) of patients with stable COPD referred for pulmonary rehabilitation were frail according to the Fried phenotype criteria. Patients who were frail demonstrated high levels of impairment as compared with prefrail or robust patients, one consequence of which was more difficulty engaging in pulmonary rehabilitation as a mainstay of their disease management. Adjusting for all known confounders, being frail was associated with over double the odds of programme non-completion. Nonetheless, in those who completed rehabilitation there was a 
Table 1 Baseline characteristics and progression through pulmonary rehabilitation for the total cohort and stratified by frailty status

\begin{tabular}{|c|c|c|c|c|c|}
\hline & $\begin{array}{l}\text { All } \\
(n=816)\end{array}$ & $\begin{array}{l}\text { Not frail } \\
(n=82)\end{array}$ & $\begin{array}{l}\text { Prefrail } \\
(n=525)\end{array}$ & $\begin{array}{l}\text { Frail } \\
(n=209)\end{array}$ & p Value \\
\hline Age (years) & $69.8(9.7)$ & $67.4(8.1)$ & $69.0(9.6)$ & $72.6(10.0)^{*} \dagger$ & $<0.001$ \\
\hline Males, n (\%) & $484(59.3)$ & $49(59.8)$ & $324(61.7)$ & $110(52.6)$ & 0.08 \\
\hline \multicolumn{6}{|l|}{ Smoking status } \\
\hline Current:former:never (\%) & $17.9: 76.5: 6.6$ & $9.8: 84.1: 6.1$ & 18.7:75.0:6.3 & 15.3:77.0:7.7 & 0.45 \\
\hline $\mathrm{FEV}_{1} \%$ predicted & $48.9(21.0)$ & $57.0(22.4)$ & $49.0(20.8)$ & $46.3(20.1)^{*}$ & $<0.001$ \\
\hline MRC score & $3.3(1.1)$ & $2.4(0.8)$ & $3.2(1.0)$ & $4.0(0.9)^{*} \dagger$ & $<0.001$ \\
\hline Age-adjusted Charlson Index & $4.3(1.6)$ & $4.4(1.6)$ & $4.3(1.6)$ & $4.4(1.6)$ & 0.57 \\
\hline ADO score & $4.9(1.7)$ & $3.6(1.3)$ & $4.7(1.6)$ & $6.0(1.5)^{*} \dagger$ & $<0.001$ \\
\hline BMI (kg/m) & $27.8(6.7)$ & $27.2(5.2)$ & $27.8(6.5)$ & $27.9(7.6)$ & 0.71 \\
\hline SMI $\left(\mathrm{kg} / \mathrm{m}^{2}\right)$ & $8.47(1.87)$ & $8.6(1.9)$ & $8.6(1.9)$ & $8.1(1.8)^{*} \dagger$ & 0.002 \\
\hline Sarcopenia (\%) & $12.4(10.3,14.8)$ & $1.2(0.2,6.6)$ & $9.5(7.3,12.3)$ & $23.9(18.6,30.1)$ & $<0.001$ \\
\hline Handgrip (kg) & $27.0(9.9)$ & $33.0(8.9)$ & $28.3(9.6)$ & $21.3(8.2)^{*} \dagger$ & $<0.001$ \\
\hline Peak QMVC (kg) & $26.2(10.0)$ & $31.0(10.1)$ & $27.3(9.6)$ & $21.0(9.0)^{*} \dagger$ & $<0.001$ \\
\hline QMVC \% predicted & $59.1(20.0)$ & $67.2(20.6)$ & $60.6(20.0)$ & $51.1(16.9)^{*} \dagger$ & $<0.001$ \\
\hline Below QMVC cut-point (\%) & $25.9(22.8,29.2)$ & $6.4(2.8,14.1)$ & $21.4(18.0,25.3)$ & $44.0(36.8,51.6)^{*} \dagger$ & $<0.001$ \\
\hline 4MGS (m/s) & $0.90(0.24)$ & $1.11(0.21)$ & $0.95(0.19)$ & $0.66(0.20)^{*} \dagger$ & $<0.001$ \\
\hline ISWT (m) & $222.3(151.3)$ & $375.4(168.3)$ & $245.1(135.4)$ & $105.0(89.5)^{*} \dagger$ & $<0.001$ \\
\hline CRQ dyspnoea score & $13.9(5.6)$ & $16.4(5.9)$ & $14.0(5.7)$ & $12.7(5.0)^{*} \dagger$ & $<0.001$ \\
\hline CRQ fatigue score & $13.4(5.3)$ & $17.6(4.3)$ & $13.8(5.2)$ & $10.9(4.6)^{*} \dagger$ & $<0.001$ \\
\hline CRQ emotional score & $30.6(9.5)$ & $36.1(7.7)$ & $31.3(9.1)$ & $26.6(9.5)^{*} \dagger$ & $<0.001$ \\
\hline CRQ mastery score & $17.6(6.0)$ & $21.7(4.6)$ & $18.0(5.8)$ & $15.0(5.7)^{\star} \dagger$ & $<0.001$ \\
\hline Self-reported weekly energy expenditure (kcal) & $698.9(1478.1)$ & $1878.4(1631.0)$ & $1110.5(1549.7)$ & $257.2(450.0)^{*} \dagger$ & $<0.001$ \\
\hline Self-reported time in moderate activity (min/week) & $279.9(431.1)$ & $532.8(491.7)$ & $322.6(465.0)$ & $73.6(1292)^{*} \dagger$ & $<0.001$ \\
\hline CAT score & $20.7(8.3)$ & $13.3(5.6)$ & $20.2(7.8)$ & $25.0(7.9)^{*} \dagger$ & $<0.001$ \\
\hline Katz score & $5.7(0.7)$ & $5.9(0.2)$ & $5.8(0.5)$ & $5.4(1.0)^{*} \dagger$ & $<0.001$ \\
\hline HADS anxiety & $7.1(4.6)$ & $5.2(3.4)$ & $6.8(4.4)$ & $8.3(5.2)^{*}+$ & $<0.001$ \\
\hline HADS depression & $6.6(3.8)$ & $4.1(2.7)$ & $6.3(3.7)$ & $8.2(4.0)^{*} \dagger$ & $<0.001$ \\
\hline Self-reported hospital admission in previous year, n (\%) & $337(41.3)$ & $28(34.1)$ & $215(41.0)$ & $94(45.0)^{*} \dagger$ & $<0.001$ \\
\hline Self-report number of exacerbations in previous year & $2.8(3.6)$ & $2.1(2.3)$ & $2.8(3.9)$ & $3.1(3.0)^{*}$ & $<0.001$ \\
\hline \multicolumn{6}{|l|}{ Frailty characteristic ( $\%$ meeting criteria) } \\
\hline Unintentional weight loss & $14.2(12.0$ to 16.8$)$ & 0 & $11.8(9.3$ to 14.9$)$ & 25.4 (19.9 to 31.7$)$ & $<0.001$ \\
\hline Exhaustion & $65.3(62.0$ to 68.5$)$ & 0 & 68.4 (64.3 to 72.2$)$ & 83.3 (77.6 to 87.7$)$ & $<0.001$ \\
\hline Low physical activity & 35.9 (32.7 to 39.3$)$ & 0 & 23.8 (20.4 to 27.6$)$ & 80.4 (74.5 to 85.2$)$ & $<0.001$ \\
\hline Slow gait speed & $24.4(21.6$ to 27.4$)$ & 0 & 9.1 (7.0 to 11.9$)$ & 72.2 (65.8 to 77.9 ) & $<0.001$ \\
\hline Weak handgrip strength & 43.6 (40.3 to 47.1$)$ & 0 & 35.8 (31.8 to 40.0$)$ & 80.4 (74.5 to 85.2 ) & $<0.001$ \\
\hline \multicolumn{6}{|l|}{ Pulmonary rehabilitation } \\
\hline Started (\% of referred) & 84.7 (82.0 to 87.0$)$ & $80.4(94.1)$ & $87.4(84.3$ to 90.0$)$ & 76.1 (69.9 to 81.4$)$ & $<0.001$ \\
\hline Number of sessions attended & $11.4(4.2)$ & $13.2(3.0)$ & $11.6(4.1)$ & $10.2(4.7)$ & $<0.001$ \\
\hline Completed (\% of starters) & 83.1 (80.1 to 85.7 ) & 94.5 (86.7 to 97.8$)$ & 82.8 (79.1 to 86.0$)$ & 72.3 (64.9 to 78.7$)$ & $<0.001$ \\
\hline Completed (\% of referred) & 70.3 (67.1 to 73.4$)$ & 84.1 (74.7 to 90.5$)$ & 74.3 (70.4 to 77.8$)$ & 55.0 (48.2 to 61.6$)$ & $<0.001$ \\
\hline
\end{tabular}

Values are mean (SD) or proportions $(95 \% \mathrm{Cl})$ unless stated.

* Statistically different to non-frail group.

tStatistically different to prefrail group.

4MGS, 4-m gait speed; ADO, age, dyspnoea, and airflow obstruction; BMI, body mass index; CAT, COPD Assessment Test; CRQ, Chronic Respiratory Disease Questionnaire; HADS,

Hospital Anxiety and Depression scale; ISWT, incremental shuttle walk test; kcal, kilocalorie; MRC, Medical Research Council; QMVC, quadriceps maximum voluntary contraction; SMI,

skeletal muscle mass index.

gradient of treatment response, in favour of patients who were frail, for outcomes relating to symptom burden, exercise performance and health status. Furthermore, in a large proportion of those who completed pulmonary rehabilitation, the frailty phenotype was reversed, at least in the short term.

Our point prevalence estimate for frailty is within the range previously reported in COPD (10.2\% to $28.0 \%),{ }^{5737}$ and over twice the $9.9 \%$ (95\% CI $9.6 \%$ to $10.2 \%$ ) observed among older people living in the community. ${ }^{2}$ Prevalence in our cohort, with a mean age of 70 years, is similar to that found among those aged 85 and above in the general population. ${ }^{2}$ The only estimate to suggest frailty prevalence is not increased in respiratory disease arose from a retrospective analysis of an older-persons cohort and included patients with very mild spirometric disease (mean $\mathrm{FEV}_{1} 79.6$ (25.2) \% predicted) and minimal evidence of functional impairment (only $2.5 \%$ of patients with COPD had a slow gait speed). ${ }^{5}$ While frailty was more common in patients with spirometrically advanced disease, prevalence among patients in the GOLD I category was almost $20 \%$ emphasising the importance of multidimensional assessment, even in early disease.

The detailed phenotypic data highlight the extent to which frailty adversely impacts patients with chronic respiratory disease. Others have associated frailty with poor exercise capacity and physical disability. ${ }^{7} 38$ We extend these findings by 


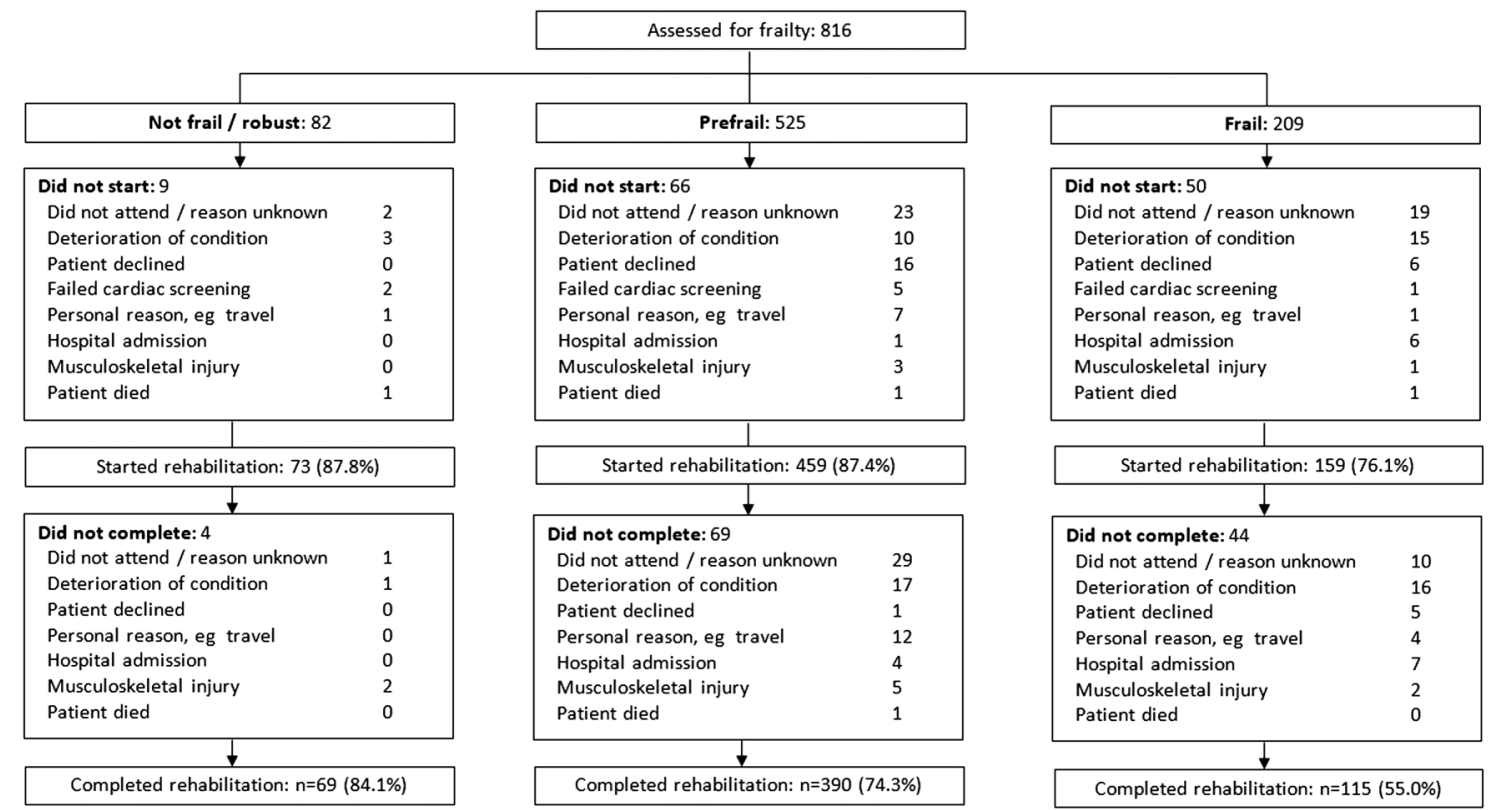

Figure 1 Profile showing recruitment, frailty status and flow of patients through the trial with reasons for non-uptake or non-completion of pulmonary rehabilitation.

demonstrating that frailty relates to reduced physical function (lower limb muscle strength, exercise capacity, physical activity), dyspnoea, dependency, increased anxiety and depression, and worse emotional distress and health status. These deficits have also been linked to sarcopenia, ${ }^{15}$ which is considered a component of frailty. ${ }^{39}$ Frailty is more multifaceted and can occur without skeletal muscle dysfunction, for example, due to ventilatory impairment, as confirmed by the only partial overlap we observed between these two syndromes. ${ }^{40}$

Irrespective of cause, frailty denotes an increased state of risk related to falls, disability, hospitalisation and mortality. ${ }^{3}$ The prognostic utility of frailty in chronic respiratory disease for mortality and hospitalisation is supported by earlier studies, ${ }^{5} 71$ and its adverse impact on the clinical management
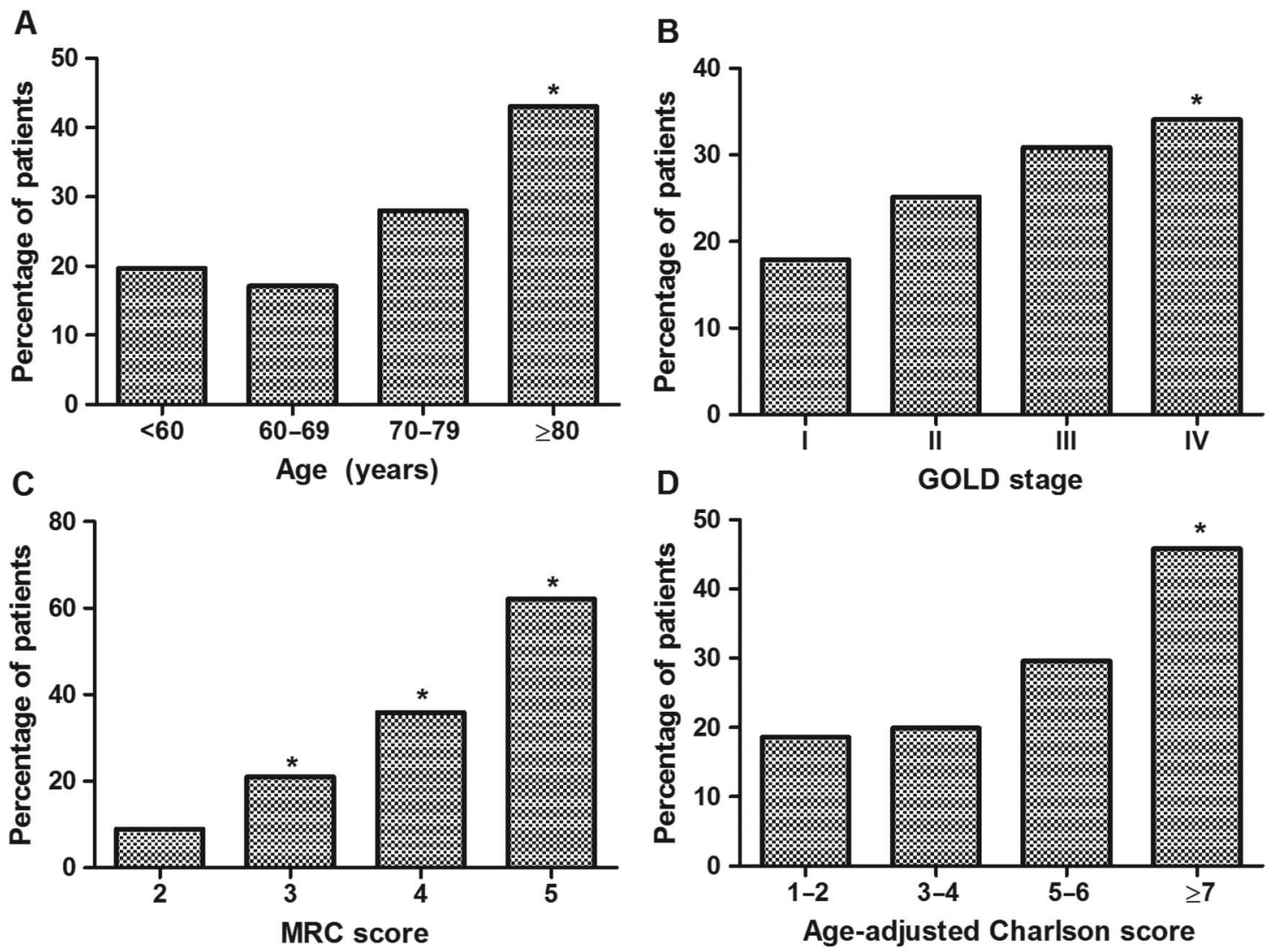

Figure 2 Prevalence of frailty in COPD according to age (A), GOLD spirometric stage (B), Medical Research Council (MRC) Dyspnoea score (C) and co-morbidity burden (D) $(n=816)$. Between-group differences $(p<0.01)$ compared with base group (far left) denoted by asterisk. 
Table 2 Univariate and multivariate logistic regression for variables associated with non-completion of pulmonary rehabilitation in patients with COPD $(n=816)$

\begin{tabular}{|c|c|c|}
\hline Univariate & OR $(95 \% \mathrm{Cl})$ & p Value \\
\hline Age & 0.983 (0.967 to 0.998$)$ & 0.029 \\
\hline Sex & 0.855 (0.628 to 1.164$)$ & 0.32 \\
\hline Current smoker (yes/no) & $1.000(1.000$ to 1.000$)$ & 0.52 \\
\hline MRC & 1.619 (1.388 to 1.887$)$ & $<0.001$ \\
\hline $\mathrm{FEV}_{1} \%$ predicted & 0.995 (0.987 to 1.002$)$ & 0.15 \\
\hline GOLD stage & $1.000(1.000$ to 1.000$)$ & 0.52 \\
\hline Age-adjusted Charlson Index & $0.926(0.849$ to 1.019$)$ & 0.11 \\
\hline ISWT distance & 0.996 (0.995 to 0.998$)$ & $<0.001$ \\
\hline QMVC & $0.986(0.970$ to 1.003$)$ & 0.12 \\
\hline ADO & $1.122(1.022$ to 1.231$)$ & 0.015 \\
\hline CAT score & 1.059 (1.038 to 1.080$)$ & $<0.001$ \\
\hline HAD anxiety & 1.061 (1.027 to 1.096$)$ & $<0.001$ \\
\hline HAD depression & $1.093(1.051$ to 1.137$)$ & $<0.001$ \\
\hline \multicolumn{3}{|l|}{ Frailty characteristic } \\
\hline Unintentional weight loss & 1.578 (1.0044 to 2.385$)$ & 0.030 \\
\hline Exhaustion & 1.615 (1.154 to 2.261$)$ & $<0.001$ \\
\hline Low physical activity & 2.334 (1.708 to 3.189$)$ & $<0.001$ \\
\hline Slow gait speed & 2.317 (1.655 to 3.244$)$ & $<0.001$ \\
\hline Weak handgrip strength & $1.258(0.927$ to 1.709$)$ & 0.141 \\
\hline Frailty status (not or pre-frail/frail) & 2.699 (1.936 to 3.762$)$ & $<0.001$ \\
\hline Multivariate & Adjusted OR (95\% CI) & $p$ Value \\
\hline Age & 0.964 (0.944 to 0.984$)$ & 0.001 \\
\hline ISWT distance & $0.998(0.997$ to 1.000$)$ & 0.026 \\
\hline CAT score & 1.024 (0.998 to 1.051$)$ & 0.07 \\
\hline Frailty status, yes/no & 2.195 (1.392 to 3.463$)$ & 0.001 \\
\hline
\end{tabular}

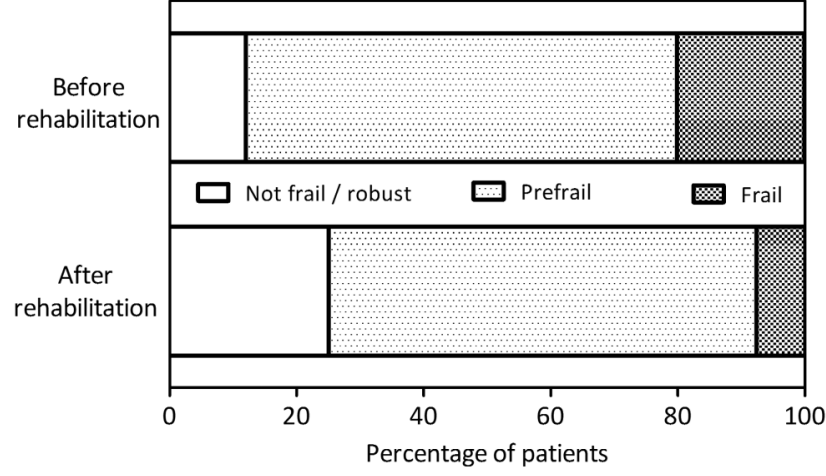

Figure 3 Patients with COPD grouped according to Fried's frailty criteria before and after pulmonary rehabilitation $(n=574)$. Overall, rehabilitation led to a shift away from physical frailty towards a more robust state.

of patients is emerging. ${ }^{7}$ Here, we highlight a new example of frailty altering COPD management in that it prevented patients from fully engaging in pulmonary rehabilitation, an internationally recommended standard of care. ${ }^{11}$ Patients who were frail experienced frequent episodes of clinical deterioration and/or hospitalisation, which restricted their participation in rehabilitation. Although previous studies have identified risk factors for pulmonary rehabilitation non-completion, for example, smoking status, breathlessness or low mood, ${ }^{17} 18$ frailty was comparatively a far stronger independent predictor in our cohort.

The impressive outcomes seen following programme completion provide strong grounds to explore how better to support patients who are frail through rehabilitation, potentially through organisation changes or by supplementing supervised exercise with novel strategies, for example, neuromuscular electrical

Table 3 Comparison of clinical outcomes following pulmonary rehabilitation according to frailty status

\begin{tabular}{|c|c|c|c|c|}
\hline & $\begin{array}{l}\text { Not frail } \\
(n=69)\end{array}$ & $\begin{array}{l}\text { Prefrail } \\
(n=390)\end{array}$ & $\begin{array}{l}\text { Frail } \\
(n=115)\end{array}$ & p Value \\
\hline MRC & $0.1(-0.3$ to 0.5$)$ & $-0.5(-0.7$ to -0.4$)$ & $-1.4(-1.1 \text { to }-1.7)^{*} \dagger$ & $<0.001$ \\
\hline SMI $\left(\mathrm{kg} / \mathrm{m}^{2}\right)$ & $0.6(0.5$ to 1.1$)$ & $0.5(0.2$ to 0.7$)$ & 0.5 (0.1 to 0.8$)$ & 0.90 \\
\hline Handgrip (kg) & $-0.2(-1.2$ to 0.9$)$ & $1.2(0.8$ to 1.5$)$ & $1.6(1.0 \text { to } 2.3)^{*}$ & 0.002 \\
\hline Peak QMVC (kg) & 2.7 (1.1 to 4.3$)$ & $1.9(1.2$ to 2.5$)$ & 1.8 (0.8 to 2.7$)$ & 0.55 \\
\hline Below QMVC cut-point (\%) & $6.4(-1.4$ to 14.1$)$ & $-21.74(-17.7$ to -25.3$)$ & $-36.6(-24.8 \text { to }-46.9)^{*} \dagger$ & $<0.001$ \\
\hline 4MGS (m/s) & $0.08(0.05$ to 0.12$)$ & $0.07(0.05$ to 0.08$)$ & $0.11(0.09$ to 0.14$)$ & 0.004 \\
\hline ISWT (m) & $17.8(-21.7$ to 57.3$)$ & 51.8 (24.4 to 79.2$)$ & $145.9(108.6 \text { to } 183.2)^{*} \dagger$ & $<0.001$ \\
\hline CRQ dyspnoea score & 3.8 (1.4 to 6.2$)$ & 4.4 (3.3 to 5.4$)$ & 6.8 (5.0 to 8.5$)$ & 0.006 \\
\hline CRQ fatigue score & $-0.8(-3.2$ to 1.5$)$ & $3.1(2.1$ to 4.0$)$ & $6.1(4.6 \text { to } 7.7)^{*} \dagger$ & $<0.001$ \\
\hline CRQ emotional score & $-0.5(-2.6$ to 3.7$)$ & $4.0(2.4$ to 5.6$)$ & $8.6(5.6 \text { to } 11.5)^{*} \dagger$ & $<0.001$ \\
\hline CRQ mastery score & $0.7(-1.1$ to 2.4$)$ & 3.1 (2.1 to 4.1$)$ & $5.2(3.4 \text { to } 6.9)^{*} \dagger$ & $<0.001$ \\
\hline Self-reported weekly energy expenditure (kcal) & 1276.0 (714.1 to 1838.0$)$ & 606.2 (390.0 to 822.5 ) & 767.1 (546.4 to 987.8$)$ & 0.08 \\
\hline Self-reported time in moderate activity (min/week) & $417.5(184.7$ to 650.4$)$ & $137.0(75.2$ to 198.9$)$ & 190.3 (127.4 to 253.3$)$ & 0.006 \\
\hline CAT score & $0.4(-1.4$ to 2.1$)$ & $-1.3(-2.7$ to 0.2$)$ & $-7.3(-9.7 \text { to }-4.8)^{*} \dagger$ & $<0.001$ \\
\hline Katz score & $0.0(-0.1$ to 0.1$)$ & $0.0(-0.1$ to 0.1$)$ & $0.1(-0.1$ to 0.3$)$ & 0.73 \\
\hline HADS anxiety & $-0.3(-2.0$ to 1.4$)$ & $-1.0(-1.7$ to -0.3$)$ & $-2.8(-4.4 \text { to }-1.2)^{*}$ & $<0.001$ \\
\hline HADS depression & $0.9(-0.2$ to 2.1$)$ & $-0.8(-1.4$ to -0.1$)$ & $-2.9(-4.0 \text { to }-1.7)^{*} \dagger$ & $<0.001$ \\
\hline
\end{tabular}

Values are mean change $(95 \% \mathrm{Cl})$ pre-to-post rehabilitation.

${ }^{*}$ Statistically different to non-frail group.

tStatistically different to prefrail group-tested if ANCOVA p value $<0.003$ following Bonferroni adjustment for multiple testing.

4MGS, 4-m gait speed; ANCOVA, analysis of covariance; BMI, body mass index; CAT, COPD Assessment Test; CRQ, Chronic Respiratory Disease Questionnaire; HADS, Hospital Anxiety

and Depression scale; ISWT, incremental shuttle walk test; kcal, kilocalorie; MRC, Medical Research Council; QMVC, quadriceps maximum voluntary contraction; SMI, skeletal muscle mass index. 
stimulation. ${ }^{42}$ Improvements across physical, psychological and global health were observed after rehabilitation, often with an apparent gradient of treatment response in favour of patients who were frail (table 2). The magnitude of effect among patients who were frail is noteworthy and many improvements far exceeded minimum important differences (MID); mean change (95\% CI) ISWT $146 \mathrm{~m}$ (109 to 183)/MID $47.5 \mathrm{~m}^{43}$ and CAT score -7.3 (-9.7 to -4.8$)$ MID $-2.0 .{ }^{34}$ This was reflected in the shift away from frailty after rehabilitation shown in figure 3. In part, this reflects the working of the Fried phenotype model, which uses cut-points to define frailty, therefore in some patients a subtle improvement would 'declassify' their frail state. Nonetheless, our data validate the contemporary view of frailty as a condition that is amenable to treatment. ${ }^{3} 39$ Treatments with evidence of efficacy in frailty management include exercise, nutritional support, self-management strategies and reduction of polypharmacy. ${ }^{39}$ Many of these components have already been operationalised to be delivered as pulmonary rehabilitation, which raises the idea the model could be adapted to support frail people outside of the respiratory specialty. Indeed, tailored frailty programmes for older adults are being piloted within geriatric medicine-the aims, structure and content of which are similar to pulmonary rehabilitation. ${ }^{44}$ We believe there is an opportunity to share learning, skills and component interventions to benefit patients across settings.

There are limitations to consider. We purposefully selected the Fried model which mainly reflects physical frailty and incorporates measures that are effort dependant or rely on patient recall. However, the model has proven construct and predictive validity and is the most established criterion measure of frailty. ${ }^{3} 79$ Other frailty assessments may capture the broader experience of the syndrome, for example, cognitive, social or environmental. ${ }^{2}$ We only enrolled patients who attended for pulmonary rehabilitation assessment, and there will likely be patients with 'hidden' frailty who were referred but unable to attend due to poor mobility or cognition. Our prevalence estimate does not take this subgroup into account, but this may be counterbalanced by the omission of asymptomatic patients with COPD, who may not have been referred to hospital outpatient or pulmonary rehabilitation clinics. We did not obtain outcomes on participants declining or dropping out of rehabilitation; therefore, our findings concerning clinical response to rehabilitation should not be generalised beyond those completing a programme. As patients who were frail had the greatest levels of impairment at baseline, regression to the mean may partially accounts for the preferential response. However, this bias is likely to be small and withholding rehabilitation from a control group could be considered unethical. The differences in some outcomes between patients who were frail and not-frail were, in part, a product of the poor response for the $10 \%$ of the cohort considered robust. This was most notable for exercise capacity and health-related quality of life, which may reflect that this fitter group had better preserved exercise capacity and health status at enrolment, and therefore had alternative targets for pulmonary rehabilitation. An example might be behavioural outcomes such as daily physical activity, which increased significantly in this group and to a greater extent to the prefrail and frail groups. Finally, outcomes were obtained immediately following rehabilitation and may reflect a transient change in frailty status. The value of these changes in COPD with respect to long-term outcomes, for example, admissions and mortality, will emerge in due course.

In conclusion, frailty affects one-quarter of patients with stable COPD assessed for pulmonary rehabilitation. Frailty is an independent risk factor for programme non-completion but appears to result in favourable rehabilitation outcomes. Future research should identify how best to support patients who are frail through pulmonary rehabilitation.

Acknowledgements The authors acknowledge the Pulmonary Rehabilitation Unit at Harefield Hospital for their assistance in collecting data for this manuscript.

Contributors MM and WD-CM contributed to the conception and design of the study. SSCK, JLC, SEJ and CMN recruited patients and conducted the study. MM, SSCK, AL, MIP and WD-CM contributed to data analysis and interpretation. All authors contributed to data interpretation and drafting the manuscript for important intellectual content.

Funding This work was supported by the NIHR Respiratory Biomedical Research Unit at the Royal Brompton and Harefield NHS Foundation Trust and Imperial College, London, UK, who partly fund MIP's salary. MM is supported by the NIHR Collaboration for Leadership in Applied Health Research and Care (CLAHRC) for South London and by Cicely Saunders International. CMN is supported by a NIHR Doctoral Fellowship. WD-CM was supported by a NIHR Clinician Scientist Award, Medical Research Council (UK) New Investigator Research Grant, NIHR Clinical Trials Fellowship and by the NIHR CLAHRC for Northwest London.

Competing interests MIP has received personal reimbursement for lecturing or consultancy regarding muscle function in COPD from Novartis and Philips Respironics. He discloses institutional reimbursement for consultancy from GSK, Novartis, Regneron, Lilly, Biomarin and BI and institutional agreements to conduct research with GSK, Novartis, AZ and Philips Respironics.

Ethics approval West London (11/H0707/2) and London Camberwell St Giles (11/ LO/1780) Research Ethics Committees.

Provenance and peer review Not commissioned; externally peer reviewed.

Open Access This is an Open Access article distributed in accordance with the terms of the Creative Commons Attribution (CC BY 4.0) license, which permits others to distribute, remix, adapt and build upon this work, for commercial use, provided the original work is properly cited. See: http://creativecommons.org/licenses/ by/4.0/

\section{REFERENCES}

1 Morley JE, Abbatecola AM, Argiles JM, et al. Sarcopenia with limited mobility: an international consensus. J Am Med Dir Assoc 2011;12:403-9.

2 Collard RM, Boter $\mathrm{H}$, Schoevers RA, et al. Prevalence of frailty in community-dwelling older persons: a systematic review. J Am Geriatr Soc 2012;60:1487-92.

3 Clegg A, Young J, lliffe S, et al. Frailty in elderly people. Lancet 2013;381:752-62.

4 Donaldson AV, Maddocks M, Martolini D, et al. Muscle function in COPD: a complex interplay. Int J Chron Obstruct Pulmon Dis 2012;7:523.

5 Lahousse L, Ziere G, Verlinden VJ, et al. Risk of frailty in elderly with COPD: a population-based study. J Gerontol A Biol Sci Med Sci 2016;71:689-95.

6 Kon SS, Jones SE, Schofield SJ, et al. Gait speed and readmission following hospitalisation for acute exacerbations of COPD: a prospective study. Thorax 2015:70:1131-7

7 Singer JP, Diamond JM, Gries CJ, et al. Frailty phenotypes, disability, and outcomes in adult candidates for lung transplantation. Am J Respir Crit Care Med 2015;192:1325-34

8 Swallow EB, Reyes D, Hopkinson NS, et al. Quadriceps strength predicts mortality in patients with moderate to severe chronic obstructive pulmonary disease. Thorax 2007;62:115-20.

9 Fried LP, Tangen CM, Walston J, et al. Frailty in older adults: evidence for a phenotype. J Gerontol A Biol Sci Med Sci 2001;56:M146-56.

10 Fried LP, Ferrucci L, Darer J, et al. Untangling the concepts of disability, frailty, and comorbidity: implications for improved targeting and care. J Gerontol A Biol Sci Med Sci 2004;59:255-63.

11 Spruit MA, Singh SJ, Garvey C, et al. An Official American Thoracic Society/ European Respiratory Society Statement: key concepts and advances in pulmonary rehabilitation. Am J Respir Crit Care Med 2013;188:e13-64.

12 McCarthy B, Casey D, Devane D, et al. Pulmonary rehabilitation for chronic obstructive pulmonary disease. Cochrane Database Syst Rev 2015;2:CD003793.

13 Kon SSC, Canavan JL, Nolan CM, et al. The 4-metre gait speed in COPD: responsiveness and minimal clinically important difference. Eur Respir 2014; 43:1298-305.

14 Dodd JW, Hogg L, Nolan J, et al. The COPD assessment test (CAT): response to pulmonary rehabilitation: a multicentre, prospective study. Thorax 2011;66:425-9.

15 Jones SE, Maddocks M, Kon SS, et al. Sarcopenia in COPD: prevalence, clinical correlates and response to pulmonary rehabilitation. Thorax 2015;70:213-18

16 Beauchamp MK, Janaudis-Ferreira T, Parreira V, et al. A randomized controlled trial of balance training during pulmonary rehabilitation for individuals with COPD. Chest 2013;144:1803-10. 
17 Keating A, Lee A, Holland AE. What prevents people with chronic obstructive pulmonary disease from attending pulmonary rehabilitation? A systematic review. Chron Respir Dis 2011;8:89-99.

18 Selzler AM, Simmonds L, Rodgers WM, et al. Pulmonary rehabilitation in chronic obstructive pulmonary disease: predictors of program completion and success. COPD 2012;9:538-45.

19 Rabe KF, Hurd S, Anzueto A, et al. Global strategy for the diagnosis, management, and prevention of chronic obstructive pulmonary disease: GOLD executive summary. Am J Respir Crit Care Med 2007;176:532-55.

20 Bolton CE, Bevan-Smith EF, Blakey JD, et al. British Thoracic Society guideline on pulmonary rehabilitation in adults. Thorax 2013;68(Suppl 2):ii1-30.

21 Radloff LS. The CES-D scale: a self report depression scale for research in the general population. Appl Psychol Meas 1977;1:385-401.

22 Garcia-Aymerich J, Farrero E, Félez MA, et al. Risk factors of readmission to hospital for a COPD exacerbation: a prospective study. Thorax 2003;58:100-5.

23 Kon SS, Patel MS, Canavan JL, et al. Reliability and validity of 4-metre gait speed in COPD. Eur Respir J 2013;42:333-40.

24 Janssen I, Heymsfield SB, Baumgartner RN, et al. Estimation of skeletal muscle mass by bioelectrical impedance analysis. J App/ Physiol 2000;89:465-71.

25 Cruz-Jentoft AJ, Baeyens JP, Bauer JM, et al. Sarcopenia: European consensus on definition and diagnosis: Report of the European Working Group on Sarcopenia in Older People. Age Ageing 2010;39:412-23.

26 Maltais F, Decramer M, Casaburi R, et al. An Official American Thoracic Society/ European Respiratory Society Statement: Update on Limb Muscle Dysfunction in Chronic Obstructive Pulmonary Disease. Am J Respir Crit Care Med 2014;189: e15-62.

27 Seymour JM, Spruit MA, Hopkinson NS, et al. The prevalence of quadriceps weakness in COPD and the relationship with disease severity. Eur Respir J 2010;36:81-8.

28 Canavan JL, Maddocks M, Nolan CM, et al. Functionally Relevant Cut Point for Isometric Quadriceps Muscle Strength in Chronic Respiratory Disease. Am J Respir Crit Care Med 2015;192:395-7.

29 Singh SJ, Morgan MD, Scott S, et al. Development of a shuttle walking test of disability in patients with chronic airways obstruction. Thorax 1992;47:1019-24.

30 Charlson ME, Pompei P, Ales KL, et al. A new method of classifying prognostic comorbidity in longitudinal studies: development and validation. J Chronic Dis 1987;40:373-83.
31 Katz S. Assessing self-maintenance: activities of daily living, mobility, and instrumental activities of daily living. J Am Geriatr Soc 1983;31:721-7.

32 Puhan MA, Garcia-Aymerich J, Frey M, et al. Expansion of the prognostic assessment of patients with chronic obstructive pulmonary disease: the updated BODE index and the ADO index. Lancet 2009;374:704-11.

33 Williams JE, Singh SJ, Sewell L, et al. Development of a self-reported Chronic Respiratory Questionnaire (CRQ-SR). Thorax 2001;56:954-9.

34 Kon SS, Canavan JL, Jones SE, et al. Minimum clinically important difference for the COPD Assessment Test: a prospective analysis. Lancet Respir Med 2014;2:195-203.

35 Revill SM, Morgan MD, Singh SJ, et al. The endurance shuttle walk: a new field test for the assessment of endurance capacity in chronic obstructive pulmonary disease. Thorax 1999;54:213-22.

36 Sewell L, Singh SJ, Williams JE, et al. How long should outpatient pulmonary rehabilitation be? A randomised controlled trial of 4 weeks versus 7 weeks. Thorax 2006;61:767-71.

37 Mittal N, Raj R, Islam EA, et al. The frequency of frailty in ambulatory patients with chronic lung diseases. J Prim Care Community Health 2016;7:10-15.

38 Park SK, Richardson CR, Holleman RG, et al. Frailty in people with COPD, using the National Health and Nutrition Evaluation Survey dataset (2003-2006). Heart Lung 2013;42:163-70.

39 Morley JE, Vellas B, van Kan GA, et al. Frailty consensus: a call to action. J Am Med Dir Assoc 2013;14:392-7.

40 Mijnarends DM, Schols JM, Meijers JM, et al. Instruments to assess sarcopenia and physical frailty in older people living in a community (care) setting: similarities and discrepancies. J Am Med Dir Assoc 2015;16:301-8.

41 Vaz Fragoso CA, Enright PL, McAvay G, et al. Frailty and respiratory impairment in older persons. Am J Med 2012;125:79-86.

42 Maddocks M, Nolan CM, Man WD, et al. Neuromuscular electrical stimulation to improve exercise capacity in patients with severe COPD: a randomised double-blind, placebo-controlled trial. Lancet Respir Med 2016;4:27-36.

43 Singh SJ, Puhan MA, Andrianopoulos V, et al. An official systematic review of the European Respiratory Society/American Thoracic Society: measurement properties of field walking tests in chronic respiratory disease. Eur Respir J 2014;44:1447-78.

44 Clegg A, Barber S, Young J, et al. The Home-based Older People's Exercise (HOPE) trial: a pilot randomised controlled trial of a home-based exercise intervention for older people with frailty. Age Ageing 2014;43:687-95. 